\title{
Fourth Order Gravity, Scalar-Tensor-Vector Gravity, and Galaxy Rotation Curves
}

\author{
Priti Mishra and Tejinder P. Singh \\ Department of Astronomy and Astrophysics, \\ Tata Institute of Fundamental Research, \\ Homi Bhabha Road, Mumbai - 400005, Maharashtra, India
}

\begin{abstract}
The Lambda-CDM model is the best fit to cosmological data, and to the observed galactic rotation curves. However, in the absence of a direct detection of dark matter one should explore theories such as MOND, and perhaps also modified gravity theories like fourth order gravity and Scalar-TensorVector Gravity [STVG] as possible explanations for the non-Keplerian behaviour of galaxy rotation curves. STVG has a modified law for gravitational acceleration which attempts to fit data by fixing two free parameters. We show that, remarkably, the biharmonic equation which we get in the weak field limit of the field equations in a fourth order gravity theory implies a modification of Newtonian acceleration which is precisely of the same repulsive Yukawa form as in the STVG theory, and the corrections could in principle be large enough to try and explain the observed rotation curves. We also explain how our model provides a first principles understanding of MOND. We also show that STVG and fourth order gravity predict an acceleration parameter $a_{0}$ whose value is of the same order as in MOND.
\end{abstract}

\section{Introduction}

In spiral galaxies the observed rotation curve profiles are strongly inconsistent with those predicted in Newtonian gravity and Galilean acceleration from the distribution of the "luminous" matter we detect. Dark matter is regarded as the most plausible explanation for the observed galaxy rotation curves. It is also strongly indicated via its pivotal role in the formation of large scale structures in the Universe, providing us with the standard $\Lambda$-CDM model.

However, until direct detection of one or more dark matter candidates takes place in the laboratory or through astronomical observations, it is perhaps useful to consider modified theories of gravity as alternate explanations for the observed galaxy rotation curves, and explore to what extent and to what accuracy they fit data. Such an analysis should be looked at in the same spirit in which modifications to general relativity such as $f(R)$ gravity are being considered as alternatives to dark energy and the cosmological constant, when it comes to explaining the acceleration of the Universe. It would be all the more appealing if a single modified gravity could account for rotation curves, cosmic acceleration and also satisfactorily explain structure formation. A preliminary attempt in this direction was made by us in [1] using a specific fourth order modified gravity. In the present article we provide details of the analysis of this fourth order theory relevant to galactic rotation curves.

Fourth order gravity theories have indeed been used before to explain rotation curves, but to the best of our knowledge, the field equations proposed by us below [and which lead to a biharmonic equation in the weak field limit] have not been considered before. Earlier works on fourth order gravity in this context include those of Stabile and Scelza 2, and Mannheim and Kazanas [3. The work 22 is based on a generalization of general relativity where the Einsten-Hilbert Lagrangian is replaced by a generic function of the Ricci scalar and the Ricci and Riemann tensors. The work in 3 is based on conformal Weyl gravity. Rotation curves in $R^{n}$ gravity have been considered in 4 . Of course the fact that nonminimally coupled theories of gravity show a modification of Poisson's equation is well-known since at least thirty years (see for example the work of [5]). 
There are various theoretical reasons [independent of the need to explain rotation curves or cosmic acceleration] for considering alternatives to general relativity [GR], including higher derivative theories of gravity. GR admits gravitational singularities, which are possibly avoided in a quantum theory of gravity. Effective quantum corrections arising from quantum gravity theories can generically be interpreted as higher derivative corrections to GR. Furthermore, higher derivative theories are generally better behaved in the ultra-violet regime and allow for an improved possibility of constructing a singularity free gravity theory [6]. Higher order corrections to GR also arise from considerations of unification of interactions 7].

The form of field equations considered by us below is motivated by [though, is independent of] a study of averaging of microscopic Einstein equations over a gravitationally polarized region, wherein fourth order effective corrections to Einstein equations appear owing to the existence of an underlying quadrupole moment in the mass distribution [8], 9]. Presently however, we regard our fourth order equations as arising not from averaging over moments, but as an effective classical relic of an underlying quantum theory of gravity.

We first present the field equations and their solution in the weak field limit. Next, we take recourse to an earlier tentative solution for the rotation curves problem suggested by Moffat and collaborators in their Scalar-Tensor-Vector gravity, and show that this solution holds for our model too. Lastly, we compare our work with the solution for rotation curves proposed in MOND.

\section{Fourth order gravity and the biharmonic equation for the potential}

A fourth order modified gravity has been postulated in 1 as a common explanation for the observed cosmic acceleration and for galaxy rotation curves. Here we specialize the theory to the case of galactic dynamics and give the details of the analysis pertinent to the structure of the rotation curves. The modified gravity is assumed to be described by the following effective field equations

$$
R^{\mu \nu}-\frac{1}{2} g^{\mu \nu} R=\frac{8 \pi G}{c^{4}} T^{\mu \nu}+k^{-2} R_{; \alpha \beta}^{\mu \alpha \nu \beta}
$$

where $k$ is a positive constant with dimensions of inverse length.

We shall be interested in the Newtonian weak-field limit of the above equations, so that we choose the metric to be

$$
d s^{2}=\left(1+\frac{2 \Phi}{c^{2}}\right) c^{2} d t^{2}-d x^{2}-d y^{2}-d z^{2}
$$

and the only non-zero component of the energy-momentum tensor is $T_{0}^{0}=c^{2} \mu(r)$ where $\mu(r)$ is the matter density distribution.

In this limit the modified field equations (1) reduce to the following fourth order biharmonic equation, which is a modification of the Poisson equation

$$
\nabla^{4} \Phi-k^{2} \nabla^{2} \Phi=-4 \pi G k^{2} \mu(r)
$$

It will be shown that this fourth order biharmonic modification of the Poisson equation explains the observed galaxy rotation curves without dark matter.

We are interested here in the modification of the radial dependence of the potential. Using separation of variables, the radial part of this equation is given by:

$$
\phi^{\prime \prime \prime \prime}+\frac{4}{r} \phi^{\prime \prime \prime}-k^{2} \phi^{\prime \prime}-\frac{2}{r} k^{2} \phi^{\prime}=-4 \pi G k^{2} \mu(r)
$$

where a prime denotes a derivative with respect to $r$.

We find the series solution of this equation using the standard Frobenius method around the regular singular point $r=0$.

Case 1: The vacuum solution $\mu=0$ [homogeneous equation]: In this case we get the following solution for the acceleration $a=-\nabla \phi$

$$
a(r)=-\left(C_{0}+\frac{C_{1}}{k}\right) \frac{e^{k r}}{2 k r}+\left(C_{0}-\frac{C_{1}}{k}\right) \frac{e^{-k r}}{2 k r}+\left(C_{0}+\frac{C_{1}}{k}\right) \frac{e^{k r}}{2 k^{2} r^{2}}+\left(C_{0}-\frac{C_{1}}{k}\right) \frac{e^{-k r}}{2 k^{2} r^{2}}-\frac{C_{2}}{r^{2}}
$$


Since we have assumed $k>0$, this implies that in Eqn. (5), terms proportional to $e^{k r} \rightarrow \infty$ as $r \rightarrow \infty$ which is unphysical. So we set the coefficient of $e^{k r}$ to zero. Thus

$$
\left(C_{0}+\frac{C_{1}}{k}\right)=0 \Rightarrow C_{1}=-k C_{0} .
$$

Hence

$$
\begin{aligned}
& a=C_{0} \frac{e^{-k r}}{k r}+C_{0} \frac{e^{-k r}}{k^{2} r^{2}}-\frac{C_{2}}{r^{2}} \\
& a=-\frac{C_{2}}{r^{2}}+C_{0} \frac{e^{-k r}}{k^{2} r^{2}}(1+k r)
\end{aligned}
$$

Eqn. (8) is the solution for acceleration for the vacuum [homogeneous] case. The constants $C_{0}$ and $C_{2}$ can be related by the following reasoning: For $k r \ll 1$ we assume Newton's law of gravitation to hold, so that $C_{2}=G M+C_{0} / k^{2} \equiv G_{\infty} M$ where $G_{\infty}=G\left[1+C_{0} / k^{2} M G\right]$. For $k r \gg 1$ the exponential term can be ignored, and $G_{\infty}$ represents the effective gravitational constant at large distances. We note that overall there are two constants in the solution, $k$ and $C_{0}$, the former from the field equations, and the latter as a constant of integration.

Case 2: $\mu(r) \neq 0$ [Inhomogeneous Solution]:

Since we know the solution for the homogeneous case $(\mu=0)$, we can construct the solutions for the inhomogeneous case using the homogeneous solutions. As is well known, if $y=a u+b v$ is a solution of

$$
y^{\prime \prime}+P(x) y^{\prime}+Q(x) y=0
$$

where $a$ and $b$ are constants, we can find the solution of

$$
y^{\prime \prime}+P(x) y^{\prime}+Q(x) y=R(x)
$$

in the form

$$
y=A(x) u+B(x) v
$$

where

$$
A(x)=-\int \frac{v R}{W} d x, B(x)=\int \frac{u R}{W} d x
$$

where $W$ is the Wronskian

$$
W=u v^{\prime}-u^{\prime} v
$$

Here,

$$
A(r)=4 \pi G k \int r \sinh (k r) \mu(r) d r+a_{1}, B(r)=-4 \pi G k^{2} \int r \cosh (k r) \mu d r+a_{2}
$$

Hence inside a medium with a density profile $\mu(r)$ the acceleration is given by

$$
\begin{gathered}
a=-\phi^{\prime}=-\frac{1}{r^{2}} \int r^{2}\left[\frac{4 \pi G k \cosh (k r)}{r} \int r \sinh (k r) \mu d r-\right. \\
\left.\frac{4 \pi G k \sinh (k r)}{r} \int r \cosh (k r) \mu d r+a_{1} \frac{\cosh (k r)}{r}+a_{2} \frac{\sinh (k r)}{k r}\right] d r-\frac{a_{3}}{r^{2}}
\end{gathered}
$$

The last two terms in the bracket in the above equation are same as in the homogeneous case. These two will be reduced to $a_{1} e^{-k r} / k^{2} r^{2}(1+k r)$ for the same reasons as discussed before Eqn. (8). Hence Eqn. (2) becomes

$$
\begin{gathered}
a=-\phi^{\prime}=-\frac{1}{r^{2}} \int r^{2}\left[\frac{4 \pi k G \cosh (k r)}{r} \int r \sinh (k r) \mu d r-\right. \\
\left.\frac{4 \pi G k \sinh (k r)}{r} \int r \cosh (k r) \mu d r\right] d r+\frac{a_{1} e^{-k r}}{k^{2} r^{2}}(1+k r)-\frac{a_{3}}{r^{2}}
\end{gathered}
$$


Eqn. (2) gives the acceleration for any given $\mu(r)$. One can easily read off special cases from here :

Case I : $\mu=0$ :

$$
a=-\phi^{\prime}=\frac{a_{1} e^{-k r}}{k^{2} r^{2}}(1+k r)-\frac{a_{3}}{r^{2}}
$$

which is same as equation (8), as expected. Note that $a_{3}$ is related to $a_{1}$ and $k$ as mentioned below (8).

Case II: $\mu=$ constant $=\mu_{0}$ :

$$
\begin{gathered}
a=-\phi^{\prime}=-\frac{4 \pi G \mu_{0}}{r^{2}} \int\left[\frac{r^{2} k \cosh (k r)}{r}\left\{\int r \sinh (k r) d r\right\}\right] d r+ \\
\frac{4 \pi G \mu_{0}}{r} \int\left[\frac{r^{2} k \sinh (k r)}{r}\left\{\int r \cosh (k r) d r\right\}\right] d r+\frac{a_{1} e^{-k r}}{k^{2} r^{2}}(1+k r)-\frac{a_{3}}{r^{2}}
\end{gathered}
$$

or

$$
a=-\frac{4 \pi G \mu_{0}}{3} r+\frac{a_{1} e^{-k r}}{k^{2} r^{2}}(1+k r)-\frac{a_{3}}{r^{2}}
$$

Eqn. (19) gives acceleration for a medium of constant density. Once again, $a_{3}$ is related to $a_{1}$ and $k$, and the same holds for the general solution (2). So in all the cases there are two free constants, which are to be fixed by comparison with observations of galactic rotation curves.

We now need to know the solution of the integrals in the general expression (19) for the acceleration, once a density profile $\mu(r)$ is given. This is a difficult task. However, we can put to use the results discovered earlier in a modified gravity theory known as Scalar-Tensor-Vector Gravity (STVG) developed by Moffat and collaborators [10, 11, 12. It turns out that a solution for the velocity profile, found in STVG for possibly fitting galactic rotation curves, is also a solution in our case, for realistic density profiles.

Below we briefly recall how the acceleration and velocity profile in STVG is arrived at, and in the subsequent section we use the results of STVG for our fourth order gravity.

\section{Galaxy Rotation Curves in the STVG Theory}

Here we briefly recall the form of the acceleration law, as derived in STVG theory. Details can be found in [10, 11, 12. For a related recent discussion see [13].

The STVG theory includes, apart from standard gravity, a massive vector field $\phi^{\mu}$ with a coupling $\omega$ to gravity and mass $\mu$. In its most general form, STVG assumes the coupling $\omega$, the mass $\mu$ and the gravitational constant $G$ to be space-time dependent scalar fields $\omega(x), \mu(x)$ and $G(x)$ respectively. In STVG, the action is given by

$$
S=S_{\mathrm{Grav}}+S_{\phi}+S_{S}+S_{M}
$$

where

$$
\begin{gathered}
S_{\text {Grav }}=\frac{1}{16 \pi} \int d^{4} x \sqrt{-g}\left[\frac{1}{G}(R+2 \Lambda)\right], \\
S_{\phi}=-\int d^{4} x \sqrt{-g}\left[\omega\left(\frac{1}{4} B^{\mu \nu} B_{\mu \nu}+V(\phi)\right)\right],
\end{gathered}
$$

and

$$
\begin{gathered}
S_{S}=\int d^{4} x \sqrt{-g}\left[\frac{1}{G^{3}}\left(\frac{1}{2} g^{\mu \nu} \nabla_{\mu} G \nabla_{\nu} G-V(G)\right)\right. \\
\left.+\frac{1}{G}\left(\frac{1}{2} g^{\mu \nu} \nabla_{\mu} \omega \nabla_{\nu} \omega-V(\omega)\right)+\frac{1}{\mu^{2} G}\left(\frac{1}{2} g^{\mu \nu} \nabla_{\mu} \mu \nabla_{\nu} \mu-V(\mu)\right)\right] .
\end{gathered}
$$

Here $V(\phi)$ is the potential for the massive vector field $\phi^{\mu}$ having mass $\mu$ and coupling $\omega ; V(G), V(\omega)$ and $V(\mu)$ are the potentials associated with the three scalar fields $G(x), \omega(x)$ and $\mu(x)$, respectively, and

$$
B_{\mu \nu}=\partial_{\mu} \phi_{\nu}-\partial_{\nu} \phi_{\mu} .
$$


Next, considerable simplification is imposed on the model. By assuming that $\Lambda=0, V(\phi)=0, \omega$ and $\mu$ to be constants, and by considering the motion of a test particle coupled to gravity and to the vector field, it was shown that the law of acceleration for the field outside a spherical mass $M$ is given by

$$
a(r)=-\frac{G_{\infty} M}{r^{2}}+K \frac{\exp \left(-r / r_{0}\right)}{r^{2}}\left(1+\frac{r}{r_{0}}\right)
$$

where $G_{\infty}$ is defined to be the effective gravitational constant at infinity

$$
G_{\infty}=G\left(1+\sqrt{\frac{M_{0}}{M}}\right)
$$

and $r_{0}=1 / \mu$. Here, $M_{0}$ denotes a parameter that vanishes when $\omega=0$ and $G$ is Newton's gravitational constant. The constant $K$ is assumed to equal

$$
K=G \sqrt{M M_{0}}
$$

The choice of $K$, which determines the strength of the coupling of $B_{\mu \nu}$ to matter and the magnitude of the Yukawa force modification of weak Newtonian gravity, is based on phenomenology and $M_{0}$ is a free parameter of the theory. This particular choice of expression for $K$ ensures that for $r \ll r_{0}$ the acceleration law reduces to $a(r)=-G M / r^{2}$, as may be verified by expanding the exponential in the acceleration law (25).

By using (26), one can rewrite the acceleration in the Yukawa form

$$
a(r)=-\frac{G M}{r^{2}}\left\{1+\sqrt{\frac{M_{0}}{M}}\left[1-\exp \left(-r / r_{0}\right)\left(1+\frac{r}{r_{0}}\right)\right]\right\} .
$$

One can generalize this to the case of interior of a mass distribution by replacing the factor $G M / r^{2}$ in (28) by $G M(r) / r^{2}$ :

$$
a(r)=-\frac{G M(r)}{r^{2}}\left\{1+\sqrt{\frac{M_{0}}{M}}\left[1-\exp \left(-r / r_{0}\right)\left(1+\frac{r}{r_{0}}\right)\right]\right\} .
$$

The rotational velocity of a star $v_{c}$ is obtained from $v_{c}^{2}(r) / r=a(r)$ and is given by

$$
v_{c}=\sqrt{\frac{G M(r)}{r}}\left\{1+\sqrt{\frac{M_{0}}{M}}\left[1-\exp \left(-r / r_{0}\right)\left(1+\frac{r}{r_{0}}\right)\right]\right\}^{1 / 2} .
$$

Moffat and Brownstein [1] used the data of K and B- band luminosity, velocity, distance and redshift of these galaxies from the works of Avilla-Reese et al. [14, Balin et al. [15], Begeman et al. [16] and Bekenstein [17. Moffat and Brownstein obtained reasonably useful parametric fits to the rotation curves of 101 galaxies from this data-set - these rotation curves are available in Fig. 2 of their paper [11] (here in our paper we have used a subset of this very dataset). To our understanding, their analysis and its results, which are also arrived at by us here, come close to achieving the Universal Rotation Curve proposed by Salucci et al. [18. A good fit to a large number of galaxies has been achieved with the parameters:

$$
M_{0}=9.60 \times 10^{11} M_{\odot}, \quad r_{0}=13.92 \mathrm{kpc}=4.30 \times 10^{22} \mathrm{~cm} .
$$

In the fitting of the galaxy rotation curves for both LSB and HSB galaxies, using photometric data to determine the mass distribution $M(r)$, only the mass-to-light ratio $\langle M / L\rangle$ is employed, once the values of $M_{0}$ and $r_{0}$ are fixed universally for all LSB and HSB galaxies. Dwarf galaxies are also fitted with the parameters

$$
M_{0}=2.40 \times 10^{11} M_{\odot}, \quad r_{0}=6.96 \mathrm{kpc}=2.15 \times 10^{22} \mathrm{~cm} .
$$

One can criticize the use of different values of $M_{0}$ and $r_{0}$ for different classes of galaxies, which takes away any possibility of them being universal numbers; and instead being chosen at will to fit observations. On the one hand such criticism might appear well-justified; on the other hand a closer examination reveals the possibility of profound underlying physics whose origins are yet to be understood. The ratio $G M_{0} / r_{0}^{2}$ for HSB and LSB galaxies is the same as for dwarf galaxies, and this ratio is of the order of the observed 
cosmic acceleration $\mathrm{cH}_{0}^{-1}$ (and the MOND acceleration). The constancy of this ratio has been observed across a family of large-scale-structures (see the remarkable Fig. 7 in [20]) and formed the basis of our proposal [1] that cosmic acceleration and galactic rotation curves can be explained by the same physical origin. Also, these values of the Yukawa parameters do not violate constraints coming from solar system and laboratory data [see [19] and Fig. 8 of [20]].

We will now compare the modified acceleration law of the form Eqn. (29) with the law obtained from the fourth order gravity, and show that the above interior solution works for the fourth order case as well.

\section{Comparison with the solution in Fourth Order Gravity}

A comparison and realization of the similarity between our fourth order gravity solution and the STVG solution provides a useful hint that our solution is a useful candidate for understanding rotation curves without possibly invoking dark matter.

As noted above, in STVG the acceleration outside of a body is given by

$$
a(r)=-\frac{G_{\infty} M}{r^{2}}+\frac{K}{r^{2}} \exp \left(-\frac{r}{r_{0}}\right)\left(1+\frac{r}{r_{0}}\right)
$$

This form matches exactly with that derived in (8) for the biharmonic equation. Comparing Eqn. (8) with Eqn. (33),

$$
\begin{aligned}
C_{2} & =G_{\infty} M=G\left(1+\sqrt{\frac{M_{0}}{M}}\right) M, \\
\frac{C_{0}}{k^{2}} & =K=G \sqrt{M M_{0}} \\
\& \quad k & =\frac{1}{r_{0}} .
\end{aligned}
$$

Next, in STVG theory, the acceleration inside a spherical mass distribution takes the form

$$
a(r)=-\frac{G M(r)}{r^{2}}\left\{1+\sqrt{\frac{M_{0}}{M}}\left[1-\exp \left(-r / r_{0}\right)\left(1+\frac{r}{r_{0}}\right)\right]\right\}
$$

This is the form used to fit galaxy rotation curves. We write the above equation in the following form:

$$
a(r)=\frac{M(r)}{M} A(r)
$$

where

$$
A(r)=-\frac{G M}{r^{2}}\left\{1+\sqrt{\frac{M_{0}}{M}}\left[1-\exp \left(-r / r_{0}\right)\left(1+\frac{r}{r_{0}}\right)\right]\right\}
$$

Now one would like to check whether the biharmonic equation also gives the same form of acceleration inside a spherical mass distribution. In general this would be difficult to check; below we describe a way which suffices for our purpose.

The solution of the biharmonic equation for any given mass distribution $\mu(r)$ is given by Eqn. (2). Rewriting (2),

$$
\begin{gathered}
a(r)=-\frac{1}{r^{2}} \int\left[4 \pi k G r \cosh (k r) \int r \sinh (k r) \mu d r-\right. \\
\left.4 \pi G k r \sinh (k r) \int r \cosh (k r) \mu d r\right] d r+A(r)
\end{gathered}
$$

with

$$
a_{1}=k^{2} G \sqrt{M M_{0}}, a_{3}=G \sqrt{M M_{0}}+G M
$$

we see that there is double integral over $\mu(r)$ in the above equation. We construct a function of the acceleration and its derivatives which is independent of integrals. Such a function is

$$
\chi(r)=\beta^{\prime \prime}(r)-k^{2} \beta(r)
$$


where

$$
\beta=\frac{1}{r} \frac{d}{d r}\left[r^{2}(a(r)-A(r))\right]
$$

From the solution of the biharmonic equation it can be shown that (as expected)

$$
\chi=4 \pi G k^{2} r \mu(r)
$$

We also find $\chi(r)$ from STVG theory also using equation(38). Now

$$
\begin{gathered}
a_{S T V G}(r)=a_{b h}(r), \\
\Rightarrow \chi_{S T V G}(r)=\chi_{b h}(r)
\end{gathered}
$$

where "bh"stands for biharmonic equation. Equating $\chi_{S T V G}$ and $\chi_{b h}$, we get an equation of the form

$$
P(r) M(r)+Q(r) M^{\prime}(r)+R(r) M^{\prime \prime}(r)+S(r) M^{\prime \prime \prime}(r)+T(r)=0 .
$$

where

$$
\begin{aligned}
& P(r)=-\frac{e^{-r / r_{0}} G \sqrt{\frac{M_{0}}{M}}}{r_{0}^{4}}, \\
& Q(r)=G\left[-\frac{2}{r^{3}}+\sqrt{\frac{M_{0}}{M}}\left(-\frac{2}{r^{3}}+\frac{2 e^{-r / r_{0}}}{r^{3}}+\frac{3 e^{-r / r_{0}}}{r_{0}^{3}}+\frac{e^{-r / r_{0}}}{r r_{0}^{2}}+\frac{2 e^{-r / r_{0}}}{r^{2} r_{0}}\right)-\frac{4 \pi}{r r_{0}^{2}}\right] \\
& R(r)=G\left(\frac{2}{r^{2}}+\frac{2 \sqrt{\frac{M_{0}}{M}}}{r^{2}}-\frac{2 e^{-r / r_{0}} \sqrt{\frac{M_{0}}{M}}}{r^{2}}-\frac{3 e^{-r / r_{0}} \sqrt{\frac{M_{0}}{M}}}{r_{0}^{2}}-\frac{2 e^{-r / r_{0}} \sqrt{\frac{M_{0}}{M}}}{r r_{0}}\right) \\
& S(r)= G\left(-\frac{1}{r}-\frac{\sqrt{\frac{M_{0}}{M}}}{r}+\frac{e^{-r / r_{0}} \sqrt{\frac{M_{0}}{M}}}{r}+\frac{e^{-r / r_{0}} \sqrt{\frac{M_{0}}{M}}}{r_{0}}\right) \\
& \text { and } T(r)=-\frac{e^{-r / r_{0}} G M \sqrt{\frac{M_{0}}{M}}}{r_{0}^{4}} \cdot \\
& \qquad M(r)=\frac{1}{r^{2}} \int \mu r^{2} d r
\end{aligned}
$$

Eqn. (47) contains one integral over $\mu(r)$. Dividing equation(47) by $P(r)$ and then differentiating, we get a differential equation of the form,

$$
U(r) \mu(r)+V(r) \mu^{\prime}(r)+W(r) \mu^{\prime \prime}(r)+X(r) \mu^{\prime \prime \prime}(r)=0
$$

where

$$
\begin{aligned}
U(r) & =\left(4 e^{r / r_{0}} M \sqrt{\frac{M_{0}}{M}} \pi r_{0}\left(r+r_{0}\right)+M_{0}\left(r^{2}-6 r_{0} r+5 r_{0}^{2}\right)\right), \\
V(r) & =r_{0}\left[2 e^{r / r_{0}} M \sqrt{\frac{M_{0}}{M}} r_{0}\left(2 \pi r+r_{0}\right)+M_{0}\left(-3 r^{2}+11 r_{0} r+2\left(-1+e^{r / r_{0}}\right) r_{0}^{2}\right)\right], \\
W(r) & =r_{0}^{2}\left[e^{r / r_{0}} M \sqrt{\frac{M_{0}}{M}} r_{0}\left(r+3 r_{0}\right)+M_{0}\left(3 r^{2}+\left(-4+e^{r / r_{0}}\right) r r_{0}+3\left(-1+e^{r / r_{0}}\right) r_{0}^{2}\right)\right], \\
X(r) & =-r r_{0}^{3}\left[M_{0}\left(r-e^{r / r_{0}} r_{0}+r_{0}\right)-e^{r / r_{0}} M \sqrt{\frac{M_{0}}{M}} r_{0}\right] .
\end{aligned}
$$

The solution of Eqn. (53) will tell us for what value of $\mu(r)$, STVG theory and the biharmonic equation give the same acceleration inside a mass distribution. But it is not necessary to solve (53). Instead we take the observed density profile $\mu(r)$ for a specific galaxy type and see whether it satisfies (53).

Following [11] and known observational data, we assume the following density profile $\mu(r)$

$$
\mu(r)=\frac{3}{4 \pi r^{3}} \beta M(r)\left[\frac{r_{c}}{r+r_{c}}\right]
$$


where

$$
M(r)=4 \pi \int_{0}^{r} d r^{\prime} r^{\prime 2} \mu\left(r^{\prime}\right)=M\left(\frac{r}{r+r_{c}}\right)^{3 \beta}
$$

and

$$
\beta= \begin{cases}1 & \text { for HSB galaxies, } \\ 2 & \text { for LSB \& Dwarf galaxies. }\end{cases}
$$

The reader is referred to Section 2 of 11 for a detailed discussion of the assumptions behind the choice of the mass distribution assumed above, which is a simplified parametric model which includes the contribution coming from the stellar as well as the gaseous HI component, and the choice of an assumed $M / L$ ratio.

We have taken the data set of ten LSB, HSB and dwarf galaxy masses from Table (3) in [11. Then we plot the left hand side of Eqn. (53)

$$
S T V G-B H \equiv \frac{r_{0}}{M_{0}^{2}}\left(U(r) \mu(r)+V(r) \mu^{\prime}(r)+W(r) \mu^{\prime \prime}(r)+X(r) \mu^{\prime \prime \prime}(r)\right)
$$

after making it dimensionless using parameters $r_{0}$ and $M_{0}$. We have made the assumption that $k=1 / r_{0}$. We have taken data set of $10 \mathrm{LSB}$, HSB and dwarf galaxies and for each of them we have computed $S T V G-B H$ vs. $r$ for three ranges of $r-(0.0001-1) \mathrm{kpc},(1-100) \mathrm{kpc}$ and $(100-500) \mathrm{kpc}$. We find that for the range $1-100 \mathrm{kpc} S T V G-B H$ is very small, of the order of $10^{-4}$ or $10^{-3}$. And for other ranges $S T V G-B H$ takes high values. Since the range of length scales of interest in a galaxy lie between $1-100 \mathrm{kpc}$ and $S T V G-B H$ is very small in this range, it can be said that the solution coming from STVG theory matches with the solution of the biharmonic equation, inside the medium, for the observed density profile. In this sense, the modified acceleration law resulting from the biharmonic equation is also a candidate for explaining the observed rotation curves, from the dataset used by Moffat and Brownstein if $k$ and $M_{0}$ are assumed to take the values needed to explain observations. In future work we plan to explicitly investigate how close the predictions of the biharmonic equation can come to the Universal Rotation Curve proposed by Salucci et al. [18].

The modified gravity law (37) predicted by our fourth order gravity and the biharmonic equation is not ruled out by laboratory and solar-system tests of a Yukawa type departure from the inverse square law. This maybe seen by writing Eqn.(37) in the commonly used Yukawa form

$$
a(r)=-\frac{G_{Y} M(r)}{r^{2}}\left\{1+\alpha_{Y}\left(1+\frac{r}{\lambda}\right) e^{-r / \lambda}\right\}
$$

where

$$
\alpha=\sqrt{M_{0} / M}, \quad \alpha_{Y}=-\frac{\alpha}{1+\alpha}, \quad G_{Y}=\frac{G}{1+\alpha_{Y}}, \quad \lambda=r_{0}
$$

In our work the typical values for $\lambda$ and $\left|\alpha_{Y}\right|$ are $\lambda \approx 10^{20} \mathrm{~m}$ and $\left|\alpha_{Y}\right|$ is order unity. Various laboratory and astronomical tests have been carried out to date to constrain the values of these two parameters, which respectively measure the critical length scale over which the departure from inverse square law becomes significant, and the strength of the departure. These tests are discussed in detail by Talmadge et al. 21], by Fischbach and Talmadge (Fig. 2.13) [22, by Adelberger et al. (Fig. 4) [19] and by Moffat and Toth (Fig. 8) 20. These tests include analysis of planetary orbits and planetary precession, laboratory measurements of the gravitational constant, geophysical tests, orbital motion of the LAGEOS satellite and the moon, and Lunar Laser Ranging (anomalous perigee precession). As is evident from these discussions, and especially from Fig. 8 of $[20$ these tests do not at present reach out to the range necessary to rule out the parameter values required in our fourth order theory to explain galaxy rotation curves.

\section{Comparison of our work with MOND}

Modified Newtonian Dynamics (MOND) [23, 24] and 25] is a hypothesis that proposes a modification of Newton's law of gravity to explain the observed non-Newtonian galaxy rotation curves. In MOND, the gravitational force acting on a test particle of mass $m$ is assumed to be given by the relation

$$
F=\operatorname{ma\mu }\left(\frac{a}{a_{0}}\right)
$$


where $a$ is acceleration in Newtonian mechanics and $a_{0}$ is a new fundamental constant of nature having the value $2 \times 10^{-10} \mathrm{~ms}^{-2}$. For very small accelerations [large distances] it is assumed that

$$
\mu\left(\frac{a}{a_{0}}\right)=\frac{a}{a_{0}}
$$

whereas $\mu$ approaches the value one for accelerations encountered in the solar neighborhood. For a detailed discussion of MOND, including possible functional forms for $\mu$ see for instance [26].

Hence for large distances we will have the relation

$$
\begin{aligned}
& \frac{G M}{r^{2}}=\frac{a^{2}}{a_{0}} \\
\Rightarrow a= & \frac{\sqrt{G M a_{0}}}{r} .
\end{aligned}
$$

The virtue thus is that $a$ falls as $1 / r$ rather than $1 / r^{2}$. Thus equating $a$ to the centripetal acceleration $v^{2} / r$ we get that

$$
\frac{v^{2}}{r}=a=\frac{\sqrt{G M a_{0}}}{r} \Rightarrow v=\left(G M a_{0}\right)^{1 / 4} .
$$

With the numerical choice of $a_{0}$ made above one gets the desired value of the velocity.

As we have seen above, in MOND the law of motion under a gravitational force is modified but the law of gravity is not changed. However in our work and in STVG as well the effective law of gravitation is modified by a Yukawa potential while the law of motion is unchanged. Essentially we can say that for us

$$
F=m a=\frac{G M}{r^{2}} \frac{1}{\mu} .
$$

In our work and in STVG theory as well, the acceleration is of the form

$$
a(r)=\frac{a_{N}}{\mu}
$$

where

$$
a_{N}=\frac{G M}{r^{2}}
$$

and

$$
\frac{1}{\mu}=\frac{M(r)}{M} A(r)
$$

We will now see in what sense this form matches with MOND in the region of interest.

We have

$$
a(r)=-\frac{G M(r)}{r^{2}}\left\{1+\sqrt{\frac{M_{0}}{M}}\left[1-\exp \left(-r / r_{0}\right)\left(1+\frac{r}{r_{0}}\right)\right]\right\}
$$

Let us write,

$$
\begin{aligned}
\frac{r}{r_{0}} & =x \\
\Rightarrow a(r) & =-\frac{G M(r)}{r_{0}^{2} x^{2}}\left[1+\sqrt{\frac{M_{0}}{M}}\{1-\exp (-x)(1+x)\}\right]
\end{aligned}
$$

We consider the region $r \gtrsim r_{0}$ and we define

$$
x=1+y,
$$

where $y$ is small and $y>0$.

Hence the term in square brackets in the expression of $a(r)$ can be written as,

$$
=\left[1+\sqrt{\frac{M_{0}}{M}}\{1-\exp (-x)(1+x)\}\right]
$$






Figure 1: Correction to the inverse square law as described by Eqn. (85): the solid curve depicts the Newtonian fall-off given by the first term of (85), whereas the dashed curve depicts the correction given by the second term of (85).

$$
\begin{aligned}
& =\left[1+\sqrt{\frac{M_{0}}{M}}\{1-\exp (-1-y)(2+y)\}\right] \\
& =\left[1+\sqrt{\frac{M_{0}}{M}}\left\{1-\frac{2+y}{e} e^{-y}\right\}\right.
\end{aligned}
$$

This essentially is $1 / \mu$ in the MOND notation. (Since $y$ is very small, $e^{-y} \approx 1-y$ ) and we can write this equation as

$$
\begin{aligned}
& \cong\left[1+\sqrt{\frac{M_{0}}{M}}\left\{1-\frac{(2+y)(1-y)}{e}\right\}\right. \\
& \cong\left[1+\sqrt{\frac{M_{0}}{M}}\left\{1-\frac{(2-y)}{e}\right\}\right.
\end{aligned}
$$

Keeping terms upto only first order in $y$

$$
\cong\left[1+\sqrt{\frac{M_{0}}{M}}\left\{1-\frac{2}{e}+\frac{y}{e}\right\} .\right.
$$






Figure 2: Yukawa type term in acceleration: plot of the correction factor in Eqn. (74)

$\Rightarrow$ for $r \gtrsim r_{0}$,

$$
a(r) \approx-\frac{G M(r)}{r^{2}}\left[1+\sqrt{\frac{M_{0}}{M}}\left\{1-\frac{2}{e}+\frac{y}{e}\right\}\right] .
$$

We write this acceleration as a sum of two parts, one falling as $1 / r^{2}$ and another falling as $1 / r$ :

$$
a(r) \approx-\frac{G M(r)}{r^{2}}\left[1+\sqrt{\frac{M_{0}}{M}}\left\{1-\frac{3}{e}\right\}\right]-\frac{G M(r)}{r}\left[\sqrt{\frac{M_{0}}{M}} \frac{1}{r_{0} e}\right] .
$$

Figure 1 compares the first and second terms in the acceleration formula given above. As anticipated the second term dominates for large $r$. Also, for the acceleration to behave as $1 / r$ in the region of interest, the second term inside the curly braces in Eqn. (72) should rise linearly with $r$ - this is evident from Fig 2. The region in which the second term dominates is where the galaxy rotation curve becomes non-Newtonian. This is clear from Fig. 3, At even larger distances, the second term is exponentially damped and the fall off is again Keplerian but with an effectively larger value of $G$.

In summary it is evident that in our work the observed rotation curve arises because the Yukawa type correction dominates over the monopole term in the middle region, whereas the monopole dominates at either end.

It is significant that from our work we can give an estimate of the theoretical value of $a_{0}$ in MOND. A simplistic guess would be to construct a quantity with the dimension of acceleration from our fundamental quantities $k$ and $M_{0}$. This is nothing but

$$
\begin{aligned}
G M_{0} k^{2}=\frac{G M_{0}}{r_{0}^{2}}= & =\frac{6.67 \times 10^{-11} \times 9.60 \times 10^{11} \times 2 \times 10^{30}}{\left(13.92 \times 10^{3} \times 3.08 \times 10^{16}\right)^{2}} \\
& =3 \times 10^{-10} \mathrm{~ms}^{-2}
\end{aligned}
$$




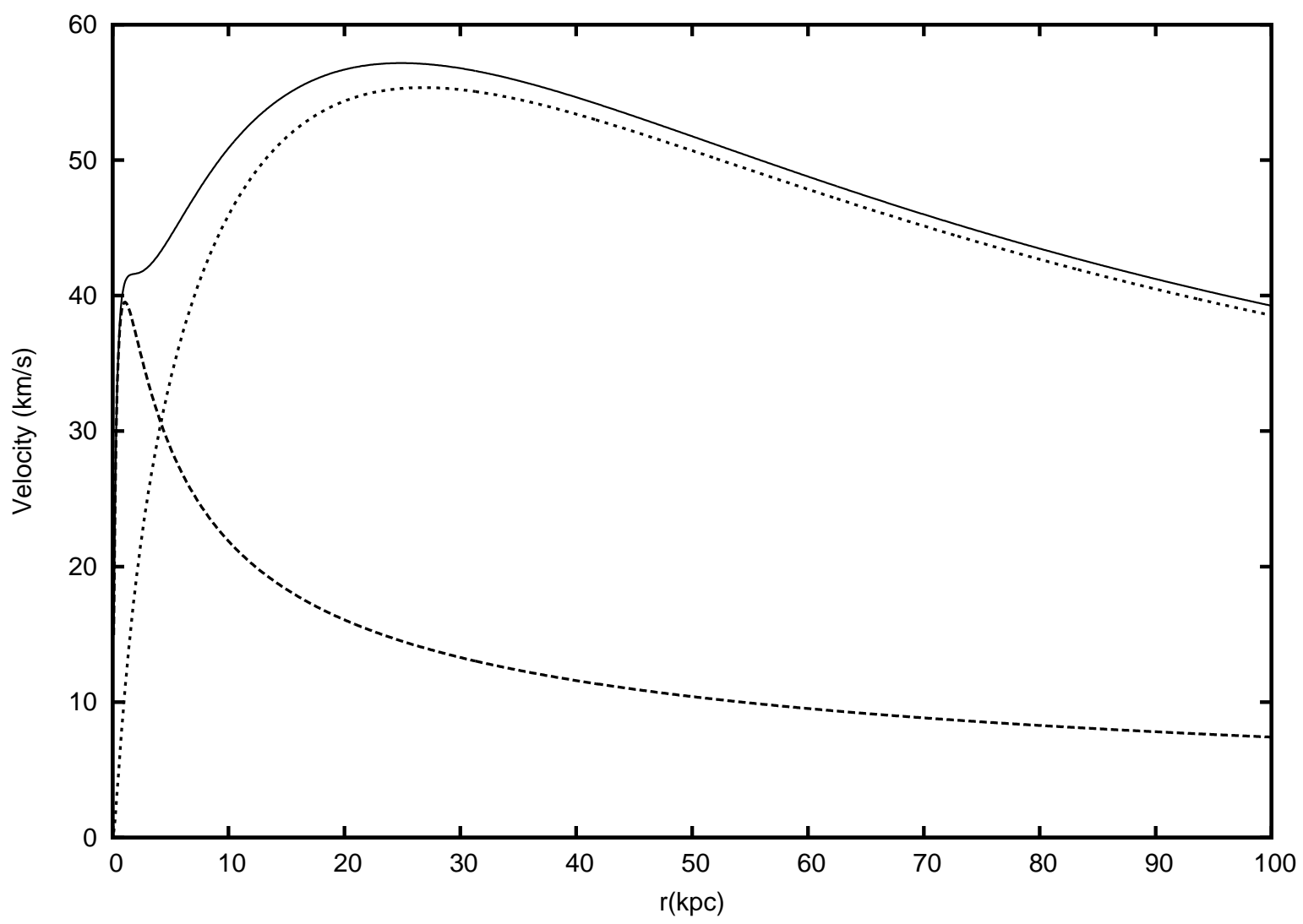

Figure 3: Plot of the velocity profile corresponding to the acceleration given by Eqn. (85). Dashed curve: velocity due to 1 st term, dotted curve velocity due to 2 nd term, thin curve: total velocity

which is of the same order as $a_{0}$ in MOND. $\left(a_{0}=2 \times 10^{-10} \mathrm{~m} / \mathrm{s}^{2}\right)$. The relation is made more transparent by comparing the acceleration given by the second term of Eqn. (85) with the acceleration in MOND in Eqn. (66). This comparison yields the fundamental relation

$$
a_{0}=\frac{G M_{0}}{r_{0}^{2} e^{2}}
$$

It shows that the parameter $a_{0}$ in MOND is of the same order as predicted from fourth order gravity. In principle then, the MOND effect can alternately be attributed to a modified fourth order gravity. The difference from MOND is that in the modified gravity case the rotation curve again falls at very large distances, whereas in MOND it continues to remain flat.

\section{Conclusions}

We found the general series solution to the biharmonic equation for the gravitational potential in the proposed fourth order gravity. We saw that the form of acceleration is very similar to that in STVG [10] in vacuum. Then we investigated further if the form of acceleration matches inside the matter distribution also. What we find is that both the solutions may be successful candidates for the observed matter density profile of galaxies. As a consequence our fourth order gravity model is capable of explaining the observed rotation curves.

The fact that the modified acceleration law discussed in this paper also arises from apparently independent considerations as in STVG and MOND, is intriguing and suggests that further investigation would be of interest. 
Acknowledgements : One of the authors (TPS) is grateful to Roustam Zalaletdinov for extensive discussions and collaboration during the early part of this work, and for telling him about the work of Szekeres, and his own work, on gravitational quadrupole polarization. The solution (8) of the biharmonic equation was found jointly with RZ, during the latter's visit to TIFR. Thanks are due to Aniket Agrawal, J. R. Brownstein, John Moffat and Viktor T. Toth for useful conversations, and also to Eric Adelberger, Marco Bruni, Patrick DasGupta, Eiichiro Komatsu and Cenalo Vaz.

\section{References}

[1] Priti Mishra and Tejinder P. Singh, Int. J. Mod. Phys. 21 (2012) 1242002.

[2] A. Stabile and G. Scelza, Phys. Rev. D. 84 (2011) 124023.

[3] P. D. Mannheim and D. Kazanas, Ap. J. 342 (1989) 635.

[4] C. F. Martins and P. Salucci, MNRAS 381 (2007) 1103.

[5] K. S. Stelle, General Relativity and Gravitation 9 (1978) 353.

[6] T. Biswas, E. Gerwick, T. Koivisto and A. Mazumdar, Phys. Rev. Lett. 108.031101 (2012)

[7] Clifford M. Will, Living Reviews Rel. 9:3 (2005)

[8] P. Szekeres, Annals of Physics 64 (1971) 599.

[9] G. Montani, R. Ruffini and R. Zalaletdinov, Class. Quantum Grav. 20 (2003) 4195.

[10] J. W. Moffat, JCAP 0603 (2006) 004.

[11] J. R. Brownstein and J. W. Moffat, Ap. J. 636 (2006) 721.

[12] J. R. Brownstein (2009) arXiv:0908.0040.

[13] J. W. Moffat and S. Rahvar (2013) arXiv:1306.6383.

[14] V. Avila-Reese, P. Colin, O. Valenzuela, E. D' Onghia, and C. Firmani, Ap. J. 559 (2001) 516

[15] J. Bailin, C. Power, B. K. Gibson, and M. Steinmetz 2005(astro-ph/0502231)

[16] K. G. Begeman, A. H. Broeils, and R. H. Sanders, MNRAS 249 (1991) 523

[17] J. D. Bekenstein, Phys. Rev. D 70 (2004) 083509

[18] P. Salucci, A. Lapi, C. Tonini, G. Gentile, I. Yegorova and U. Klein, Mon. Not. R. Astron. Soc. 378 (2007) 41

[19] E. G. Adelberger, B. R. Heckel, and A. E. Nelson, Annual Review of Nuclear and Particle Science $53(2003) 77$.

[20] J. W. Moffat and V. T. Toth, Class. Quantum Grav. 26 (2009) 085002.

[21] C. Talmadge, J.-P. Berthias, R. W. Hellings and E. M. Standish, Phys. Rev. Lett. 61 (1988) 1159

[22] E. Fischbach and C. L. Talmadge, The search for non-Newtonian gravity, Springer-Verlag (1999)

[23] M. Milgrom, Ap. J. 270 (1983) 365.

[24] M. Milgrom, Ap. J. 270 (1983) 371.

[25] M. Milgrom, Ap. J. 270 (1983) 384.

[26] J. D. Bekenstein, Contemporary Physics 47 (2006) 387. 